\title{
Differentiated chondrosarcoma originating in the cricoid cartilage of the larynx: evaluation and treatment. Clinical case presentation
}

\author{
Dr. Konstantin Borisov \\ ENT clinic at University First MHAT-Sofia EAD \\ Under the leadership of Prof. Veselin Pavlov MD
}

\begin{abstract}
Background

Modern medicine has focused much of its activity and scientific endeavours on the fight against malignant diseases. The goal of our clinical case presentation is to introduce the evaluation and treatment of a rare head and neck tumor, the laryngeal chondrosarcoma (CS). In such rare malignancies the end results of the treatment plan are associated with multiple cofactors that influence strongly the course of the disease.
\end{abstract}

\section{Materials and methods}

In our clinical case we have described the investigations and chosen tactics of treatment, applied to our patient, in a chronological manner, some immediate results, but also the long-term consequences of the management of this disease.

\section{Results}

In our opinion, the outcomes in this patient's case were prompted not only by the specifics of this malignant disease, but also by the interpretation of the instrumental examination and the decisionmaking regarding the initial treatment. In addition to that, the decisions taken by the patient also play an important role in the clinical course. We want to point out that the achieved results and outcomes could be unpredictable - due to the coincidence of circumstances - and different, in comparison to the cases described in most literature.

\section{Conclusions}

1. The relatively simple diagnostics with modern diagnostic methods and the abundance of options for surgical treatment or even combination therapy in complicated cases, do not create difficulties in the management of typical chondrosarcoma of the larynx. However, its treatment requires the efforts of several medical specialties Surgery, Medical oncology, Medical imaging, Radiation therapy, Physiotherapy and Rehabilitation, Psychology and others.

2. Neither RT nor chemotherapy are recommended therapeutic options, except in some rare individual cases.

3. Conservative surgery tends to be adequate only if performed properly and timely as thus ensures long-term remission or cancerfree life. Radical surgical treatment is recommended in advanced cases.

\section{Introduction}

Laryngeal carcinoma accounts for $1-2 \%$ of all human malignancies, but as a malignancy of the head and neck, it is the most common tumor. According to their histogenesis, malignant diseases of the larynx are divided into epithelial and mesenchymal. CS of the larynx is a rare pathology, approximately from $0.07 \%$ and up to $0.2 \%$ (or up to $1 \%$ by others) of all head and neck malignancies, although it is the most common mesenchymal origin neoplasm of the larynx. Only $2-5 \%$ of all chondrosarcomas arise in the head and neck, where the majority of them develop in the maxilla, but other typical localizations include the nasal septum, the voice box, and other parts of the skull. The most frequent location is posterior lamina's internal surface of the cricoid, almost $80 \%$ of the cases of laryngeal CS; the thyroid cartilage, especially the inferolateral wall, is affected in about $20 \%$ of cases respectively, followed by the arytenoid cartilage, the vocal cords, the hyoid bone and the epiglottis. There have also been cases when more than one cartilage is involved. In our case, the patient's tumor was located in the plate of the cricoid cartilage and the first tracheal ring.

\section{Case report}

M.P., a 62-year-old man, non-smoker, with a concomitant disease - Arterial hypertension second degree. The patient was admitted to the clinic for diagnostic clarification and treatment, with com- 
plaints of voice change, dry spastic cough and moderate shortness of breath. The complaints had been ongoing for about three months and were progressing. Physical examination: Blood pressure 138/68 mm Hg, pulse 90 beats / minute, body temperature $36.7^{\circ} \mathrm{C}$, pronounced inspiratory dyspnea, inspiratory stridor and circulation in the jugulum. Paraclinic - normal. Chest X-ray: Expanded and enlightened lung fields. There was no evidence of an active pulmonary process. The hila were unenlarged, compacted. Cardiovascular shadow - within normal limits in regard to age. Indirect laryngoscopy - a tumor formation subchordally to the right, greatly narrowing the endolarynx. We concluded that there are indications for performing an emergency tracheostomy.

After the tracheostomy, a CT scan was performed. Description: Presence of a tracheostomy. CT data of a soft tissue formation, with the presence of a coarse calcification in it, involving, two-thirds of the cricoid ring originating from right to left, which has stenosed the subglottic lumen. The described formation has a very rich blood supply. It has an axial plane size of $98.8 / 65.5 \mathrm{~mm}$. Several (more than three) lymph nodes are present bilaterally around the described formation. They have preserved fat centers and sizes up to $8.1 \mathrm{~mm}$. CT shows destruction of the cricoid cartilage on the right side. Microlaryngosurgery was performed in a planned manner. Under general anesthesia, material was taken and sent for histological examination.
Histology findings: the sample coincides with the morphology of a differentiated chondrosarcoma. After the obtainment of the histological result, total laryngectomy was performed under general anesthesia. The skin-platysma flap was incised by a "U" shaped cut of skin and subcutaneous tissue. The larynx was skeletonized and mobilized, we ligated and cut a., v., n., Laryngeus supp. bilaterally. The larynx was removed. Hemostasis. Plasticity of the pharyngostomy. Drainage, layered suture, dry sterile bandage. Intraoperative - infiltrative growth, covering the entire larynx, more to the right, without infiltration of the surrounding tissues. Histology findings: "Islands" of hyaline cartilage with increased cellularity and nuclear atypism and binuclear cells. Highly differentiated chondrosarcoma. On the 3rd postoperative day the patient stood up alone, ambulated, and had normal peristaltic. We removed the drainage. After the initial wound healing, the patient was discharged from the clinic in good general condition. We did not recommend adjuvant irradiation as a postoperative therapeutic option.

Almost a year and four months after the primary operation, the patient was admitted to the clinic with complaints of "swelling" of the neck bilaterally, more to the right, ongoing for about two months. During the native CT examination of the cervical region, large soft tissue growths were found, more pronounced on the right side with numbness in them. Multicolored soft tissue areas were scanned

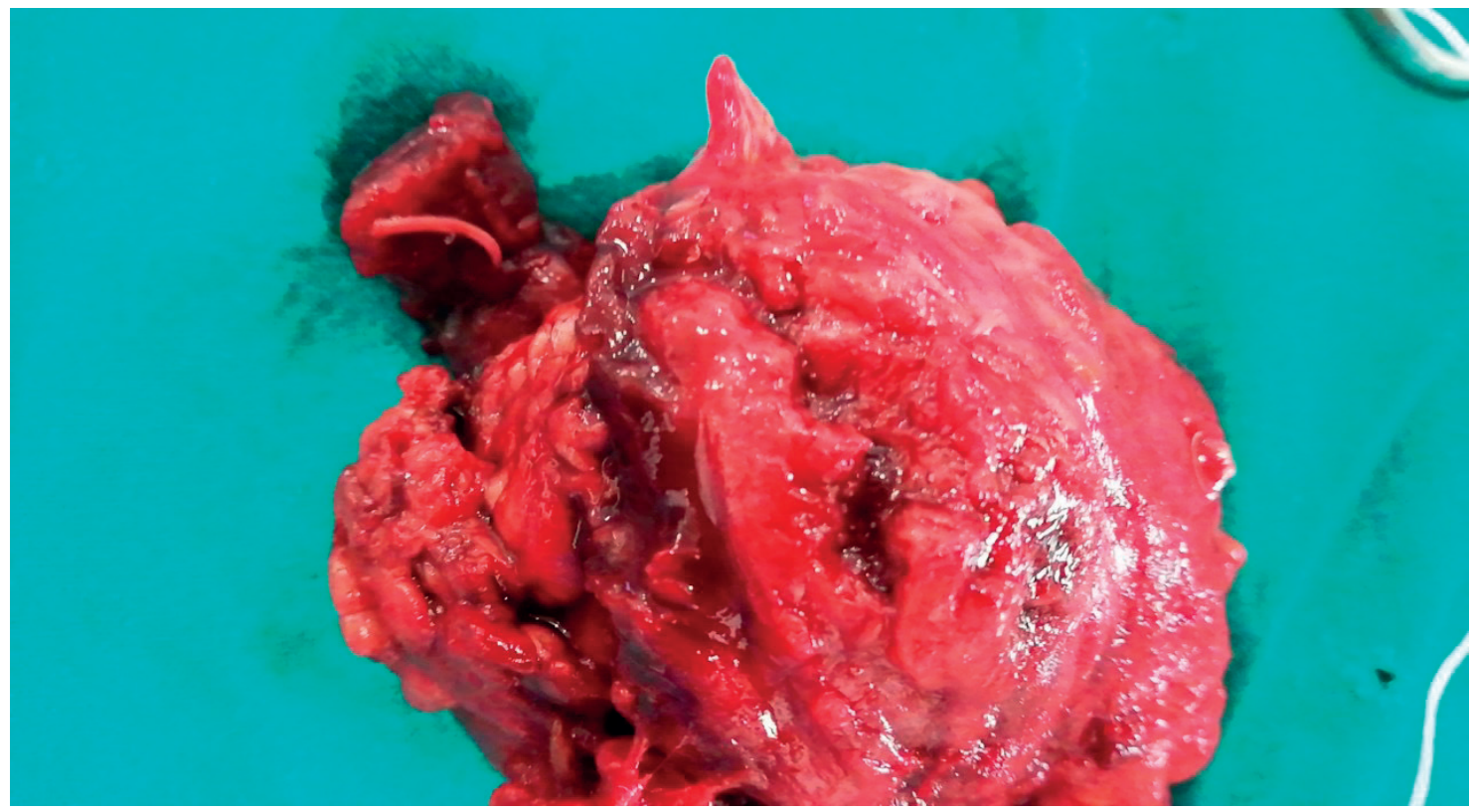

Fig. 1. Highly differentiated chondrosarcoma with infiltrative growth, covering the entire laryngeal cavity, more to the right side. 
along the course of the two mm. Sterncleidomastoidei due to enlarged lymph nodes. An incision was made on the right side of the neck under general anesthesia. Gradually, an egg-sized tumor mass, located precarotidly, was dissected and removed. A second walnut-sized tumor mass was removed from the tracheostomy area on the right. Hemostasis was performed, aspiration drainages were placed. Layered suture, dry sterile bandage. Histology findings: soft tissues with infiltration of chondrosarcoma. After a smooth postoperative period and the initial wound healing, the patient was discharged from the clinic in good general condition. To date no further progress has ever been marked in the person's record at the outpatient clinic.

\section{Discussion}

Patients with laryngeal CS exhibit a variety of symptoms as a result of tumor growth, including dyspnea, dysphagia, hoarseness, airway obstruction, even stridor, or pain, but mostly - hoarseness and difficulty in breathing. Vocal cord stiffness is observed due to the involvement of the cricoarytenoid articulation, and not because of a lesion of the recurrent nerve. Men are affected more frequently than women, usually during the middle to later decades of life. The male to female ratio is from $3: 1$ to as high as $10: 1$. Chondrosarcomas mostly occur between $40-80$ years of age with a peak in the seventh decade of life. The average age for diagnosis is usually between 64 and 66 years, thus coinciding with the maximum cartilage ossification. No definitive etiology for laryngeal CS is known, although the most commonly accepted version is an initial disordered ossification of the laryngeal cartilages. Ionizing radiation is among the etiological factors, which play a significant part. Many authors describe the evolution of the tumor against the background of multiple trauma and operations. Tobacco abuse is likely to have an influence on the development of CS, even though two thirds of the patients report not having such a habit in their medical history. Ultimately, no correlation with nicotine dependence or alcohol abuse was revealed. The transformation of chondroma to CS also deserves our attention. Ischemic changes in the chondroma due to a mechanical trauma may be a precursor to malignisation or a more aggressive biological behavior. The frequent correlation between chondroma and chondrosarcoma supports this theory. Studies reveal that it may reach $60.4 \%$ correlation rate. Irradiation is yet another therapeutic option, although according to most literture sources no effect has been found. The differential diagnosis for CS of the larynx is practically limited to chondroma, chondrometaplasia, and tracheopathia osteoplastica. Rare examples of other sarcomas have been described in the larynx and hypopharynx, such as liposarcoma, osteosarcoma, angiosarcoma, synovial sarcoma, malignant fibrous histiocytoma, Kaposi's sarcoma, leiomyosarcoma. CS are characterised by a low tendency to spread further to the regional lymph nodes, but additional agressive mesenchymal components could rarely develop in the lesion. High-grade tumours tend to be more associated with higher recurrence rate. Larger studies report an overall recurrence rate of $16 \%$ to $18 \%$. The recurrence development is more expectable if the primary neoplasm has been excised partially. The curative potential of total laryngectomy after recurrent laryngeal CS is comparable to that of initial radical surgery. Tumour-related fatal outcomes are occasional and happen when relapses with unpredictable growth or involvement of nearby greater vessels and aggressive spreading proceed. Metastases, most common to the lung, bones and liver, have been described in about $2 \%$ to $10 \%$ of the reported cases of laryngeal CS. The 5 -year survival rate is $90 \%$, while the 10 -year survival rate decreases to $80.9 \%$. The diagnosis is based on morphogical findings, and histopathology takes an exclusive role in diagnosing CS. CT scanning of the neck with contrast is a standard method for evaluating CS of the larynx, while simultaneously allowing preoperative planning. Due to the slow growing manner of this tumor, a displacement rather than invasion of the surrounding structures is observed. Ultrasound investigation can be usefull if doubtful abnormalities in the neck regions are suspected. Modern treatment guidelines assume surgery as a treatment of choice. Adequate conservative surgery must allow for eradication of the cancer within safe margins. Wide surgical excision is the principal goal. Seeking acceptable functional results always is the aim, especially when the lesion exceeds not more than half of the cricoids' perimeter, and if the histological grade is low. The extent of resection to ensure control depends on the site, size and wideness of the lesion - debulking alone, hemi- or partial laryngectomy can definitely all be adequate. Attention should be paid to the resection of the external perichondrium. Salvage 
laryngectomy becomes necessary if the cancer is larger, comes with infiltration in the surrounding tissues or an organ-sparing surgery can no longer be applied.

The role of radiotherapy is uncertain, even controversial, because these CS rarely respond to radiation treatment. Their non-responsiveness to radiation therapy limits therapeutic options. Laryngeal CS does not respond to chemotherapeutic agents.

\section{Conclusions}

The relatively simple diagnostics with modern diagnostic methods and the abundance of options for surgical treatment or even combined therapy in complex cases, do not create difficulties in the treatment of the typical chondrosarcoma of the larynx. However, their treatment requires the joint efforts of several medical specialties - Surgery, Medical oncology, Medical imaging, Radiation therapy, Physiotherapy and Rehabilitation, Psychology and others. Radiation therapy and chemotherapy are not recommended as therapeutic options, except in some rare individual cases. Conservative surgery tends to be adequate if performed properly and timely Conservative surgery tends to be adequate only if performed properly and timely as thus ensures long-term remission or cancer-free life. Radical surgical treatment is recommended in advanced cases.

\section{References}

1. Buda I, Hod R, Feinmesser R, Shvero J. Chondrosarcoma of the larynx. Isr Med Assoc J. 2012;14:681-4.

2. Sauter A, Bersch C, Lambert KL, Hömann K, Naim R. Chondrosarcoma of the larynx and review of the literature. Anticancer Res. 2007; 27: 2925-30.

3. Friedlander PL, Lyons GD. Chondrosarcoma of the larynx. Otolaryngol Head Neck Surg 2000;122:617.

4. Thompson L, Gannon F. H. Chondrosarcoma of the larynx: a clinicopathologic study of 111 cases with a review of the literature. Am J Surg Pathol. 2002; 26(7): 836-51.

5. Kardesa A, Slootweg PJ. Pathology of the head and neck. Germany: Springer-Verlag; 2006. 338 p.

6. Pocius L, Čepulis V. Acta medica Lituanica. 2012. Vol. 19. No. 4. P. 451-457

7. Berry S, Addams-Williams J, Khalil H, Armstrong S, Denton K, Baldwin D. Chondrosarcoma of the larynx. Internet J Otorhinolaryngol. 2003; 2(2): 28 .

8. Q. Wang, H. Chen, and S. Zhou, "Chondrosarcoma of the larynx: report of two cases and review of the literature," International Journal of Clinical and Experimental Pathology, vol. 8, no. 2, pp. 2068-2073, 2015.

9. Dailiana T, Nomikos P, Kapranos N, Thanos L, Papathanasiou M, Alexopoulou E, et al. Chondrosarcoma of the larynx: treatment with radiotherapy. Skeletal Radiol. 2002; 31(9): 547-9.

10. Lalwani AK. Current Diagnosis and Treatment in Otolaryngology - Head and Neck Surgery. 3rd ed. New York: McGraw Hill Medical; 2012. $1013 \mathrm{p}$.

11. Mokhtari S, Mirafsharieh A. Clear cell chondrosarcoma of the head and neck. Head Neck Oncol. $2012 ; 4: 13$.

12. Bulanov DV, Sergeev SA, Kuzmin IW, Semenov LA, Mahson AN, Bulycheva IW. Clinical and morphological features of laryngeal chondrosarcoma. Arch Pathol. 2007; 6(69): 50-2. 